\title{
Simulação de Monte Carlo para estimativa do tempo ótimo de contratos de concessão: estudo de caso baseado em rodovias do Rio Grande do Sul
}

\section{Rodrigo Nobre Fernandez}

Departamento de Economia da Universidade Federal de Pelotas (UFPEL), Pelotas, RS - Brasil

\section{André Carraro}

Departamento de Economia da Universidade Federal de Pelotas (UFPEL), Pelotas, RS - Brasil

\section{Helton Saulo}

Departamento de Estatística da Universidade de Brasília (UNB), Brasília, DF - Brasil

\section{Douglas Pivatto}

Prefeitura Municipal do Rio Grande, Rio Grande, RS - Brasil

\section{Everton Freitas}

Engenheiro de Produção

Este trabalho tem por objetivo analisar os contratos de concessão de praças de pedágio existentes no Estado do Rio Grande do Sul no período de 1998 até 2012, utilizando as informações obtidas do Relatório de Acompanhamento do Programa Estadual de Concessão Rodoviária do Rio Grande do Sul (PECR-RS). Para atingir tal meta, emprega-se o modelo de simulação proposto por $\mathrm{Ng}$ et al. (2007), o qual utiliza o procedimento de simulação de Monte Carlo, que permite inserir as informações contratuais, de modo a visualizar um possível comportamento das empresas durante a execução dos mesmos. Em suma, os resultados encontrados indicam que, para as praças analisadas com a taxa interna de retorno sendo aquela considerada no projeto básico de exploração, o tempo ótimo simulado foi igual ou menor do que aquele acordado contratualmente. Deve-se destacar que os contratos apresentados pelas concessionárias Rodo Sul e Santa Cruz seriam economicamente inviáveis dentro deste período de tempo.

Palavras-chave: concessões rodoviárias, análise de projetos, simulação de projetos 


\section{Simulación de Monte Carlo para estimar el tiempo óptimo de los contratos de concesión: estudio de caso basado en carreteras en Rio Grande do Sul}

Este trabajo tiene como objetivo analizar los contratos de concesión para las plazas de peaje existentes en el Estado de Rio Grande do Sul de 1998 a 2012, utilizando la información obtenida del informe de monitoreo del Programa Estatal de Concesión de Carreteras de Rio Grande do Sul (PECR) -RS). Para lograr este objetivo, se usa el modelo de simulación propuesto por Ng et al. (2007), que utiliza el procedimiento de simulación Monte Carlo, que permite ingresar información contractual, para visualizar un posible comportamiento de las empresas durante su ejecución. En resumen, los resultados encontrados indican que, para las plazas de peaje analizadas siendo la tasa interna de retorno considerada en el proyecto de exploración básica, el tiempo óptimo simulado fue igual o menor que el acordado en el contrato. Cabe señalar que los contratos presentados por los concesionarios Rodo Sul y Santa Cruz serían económicamente inviables dentro de este período de tiempo.

Palabras clave: concesiones de carreteras, análisis de proyectos, simulación de proyectos

Monte Carlo simulation to estimate the concession contracts optimal time: a case study based on Rio Grande do Sul highways

This work aims to analyze the concession contracts for toll plazas existing in the State of Rio Grande do Sul from 1998 to 2012, using the information obtained from the Monitoring Report of the State Highway Concession Program of Rio Grande do Sul (PECR-RS) .To achieve this goal, was used the computational model proposed by $\mathrm{Ng}$ et al. (2007), which uses the Monte Carlo simulation procedure, which allows entering contractual information, in order to visualize a possible behavior of companies during project execution. In short, the results indicate that for the analyzed plazas, with the internal rate of return being that considered in the basic exploration project, the simulated optimal time was equal to or less than that agreed in the contract. It should be noted that the contracts presented by the concessionaires Rodo Sul and Santa Cruz would be economically unfeasible within this period of time.

Keywords: road concessions, project analysis, project simulations 


\section{Introdução}

Em um país que centralizou a expansão da malha rodoviária no Governo Federal, não deixa de ser surpreendente que a primeira rodovia do Brasil foi particular, por meio de delegação. Datada do século 19, a Rodovia União da Indústria, a qual começou a ser construída em 1856 e terminada em 1861, tinha 144km de extensão. Era utilizada para atender ao ciclo do café, cruzando o Vale do Paraíba e atendendo aos municípios que por ali passavam (DUARTE, 2012).

Ainda que este tenha sido o primeiro caso, durante a maior parte do século 20 o Governo Federal passou a ser o principal responsável pela construção e manutenção das rodovias brasileiras. Tais experiências iniciaram na década de 1920. Em 1945, a União criou o fundo rodoviário nacional (FRN), destinado a financiar a construção e a manutenção das rodovias federais na época. Como uma consequência da crise da dívida externa do país, a década de 1980 foi marcada por inflação alta, baixo crescimento, dentre outros problemas, o que acabou por diminuir o investimento em diversos setores, como o rodoviário, ocasionando na deterioração da malha existente (BARBo et al., 2010; DUARTE, 2012).

Procurando melhorar a infraestrutura rodoviária brasileira, a partir da década de 1980 e, de forma mais intensificada na década de 1990, ocorreram diversos projetos de privatizações e concessões à iniciativa privada. Pinheiro (2003) observa que, entre 1992 e 2001, foi um período de mudanças significativas no setor de infraestrutura brasileiro. Conforme constatou o autor, nesse período a participação do setor privado aumentou de forma significativa em setores como o de telecomunicações, eletricidade, ferrovias, portos, rodovias, água e saneamento.

O Programa de Concessões de Rodovias Federais iniciou-se em 1993 com a Portaria no 010/1993, do Ministério dos Transportes (BARBo et al., 2010). No intuito de buscar melhorias nos mais diversos setores de infraestrutura brasileira, deve-se apontar como marco regulatório a Lei de Concessões (Lei no 8.987/1995[1]), lei a qual permitia que o governo concedesse à iniciativa privada a execução de um serviço, antes prestado pelo Poder Público, mediante processo de licitação (Lei no 8.666/1993[2] - Lei de Licitações), via concorrência, demonstrando capacidade para a execução do projeto, tendo por sua 
conta e risco e tendo o prazo determinado quando da assinatura do contrato. A partir da publicação da Lei das Concessões que foram assinados os primeiros contratos de concessão rodoviária no país.

Assim como em todo o país, a partir de 1995, no Rio Grande do Sul, foram feitas concessões rodoviárias a empresas privadas objetivando a diminuição dos gastos públicos. Tinha-se como principal meta aumentar a eficiência na prestação do serviço público e reduzir-se o gasto governamental. Nesse arranjo contratual, as empresas assumiam um investimento inicial atrelado à melhoria do sistema de transporte rodoviário, cobrando tarifas de pedágio dos consumidores finais.

A gestão dos contratos firmados com as empresas concessionárias implica na administração de interesses diametralmente opostos. Para o usuário, é pagar menos; para a concessionária, ter um contrato economicamente vantajoso que seja lucrativo ao longo do seu ciclo de vida. Contratos de longo tempo de duração naturalmente possuem riscos difíceis de se prever, tais como aumento do custo operacional, da taxa de juros, entre outros, que podem tornar o contrato em desequilíbrio financeiro, o que pode ocasionar revisões contratuais.

Dentro desse escopo, este trabalho tem por objetivo estimar o tempo ótimo de cada contrato para projetos de concessão de praças de pedágio existentes no Estado do Rio Grande do Sul no período de 1998 até 2012, calibrando com dados obtidos do Relatório de Acompanhamento do Programa Estadual de Concessão Rodoviária do Rio Grande do Sul (PECR-RS). Para realizar tal tarefa, os cálculos são feitos a partir do modelo de simulação proposto por Ng et al. (2007), que permite estimar com qual probabilidade o projeto será executado de forma exitosa dentro do período de contrato.

Em suma, os resultados indicam que, para as praças analisadas com a taxa interna de retorno sendo aquela considerada no projeto básico de exploração, o tempo ótimo foi igual ou menor àquele acordado contratualmente. Deve-se destacar que, com as propostas comerciais apresentadas pelas concessionárias Rodo Sul e Santa Cruz, os contratos seriam economicamente inviáveis dentro desse período de tempo.

Por fim, este trabalho está estruturado em cinco seções, tendo sido iniciado por esta breve introdução. Na seção dois, apresenta-se uma revisão da literatura. Na 
terceira, mostra-se o modelo de simulação de $\mathrm{Ng}$ et al. (2007) e os dados utilizados para a calibragem do modelo. Na quarta seção, tem-se a apresentação dos resultados, bem como a discussão. Encerra-se com as considerações finais.

\section{0 programa de concessões federais e estaduais}

A prática de concessão de rodovias para administração privada é bastante comum a diversos países ao redor do mundo. Conforme relatam Galilea e Medda (2010), Carpintero e Barcham (2012) e Beria et al. (2015), esse processo se intensificou no início da década de 1990 nos países emergentes, principalmente aqueles pertencentes aos continentes latino americano e europeu central e oriental. De acordo com o Private Participation in Infrastructure Database[3] (PPI), entre 1990 a 2018 foram investidos aproximadamente 119 bilhões de dólares em projetos de infraestrutura e concessões de rodovias.

No contexto dessas nações, a possibilidade de concessão de rodovias tornou-se uma alternativa viável tendo em vista que muitos governos apresentaram dificuldades em prover investimentos num patamar semelhante a décadas anteriores, mesmo que o aparato institucional precise evoluir bastante para atingir-se níveis adequados, e, assim, reduzir o grande número de renegociações contratuais (GUASCH et al., 2008).

No Brasil, após a década "perdida" de 1980, marcada por altas taxas de inflação, baixo crescimento econômico e políticas diversas para conter a pressão inflacionária houve uma expressiva queda no investimento, principalmente aquele relacionado a setores de infraestrutura. Tendo em vista essa degradação, tem-se que o investimento anual médio no setor rodoviário caiu de 5,2\% nos anos 1980 para 2,1\% do Produto Interno Bruto (PIB) no início dos anos 2000. O que agravava esse cenário é que as rodovias são responsáveis pela maior parte do transporte do volume de cargas no país (61\%). Deste modo, tornase visível que uma infraestrutura inadequada é um dos entraves ao desenvolvimento do Brasil (CNT, 2011).

Em 1995, iniciou-se o programa de concessões das rodovias federais, tendo sido licitados diretamente pelo Ministério dos Transportes seis trechos que operavam no sistema de pedágios em uma extensão total de aproximadamente $1.500 \mathrm{Km}$, e com prazos oscilando 
entre 20 e 27 anos. No período de 2007 a 2008 teve-se a segunda fase com a licitação de 3200 km e, em 2013, a terceira etapa do programa com 3900 km de rodovias licitados.

Embora a Agência Nacional de Transportes Terrestres (ANTT) tenha sido fundada em 2001, somente em 2005 esta instituição iniciou a realização de estudos acerca dos trechos a serem licitados. Tendo em vista que a regulação do setor estava melhor estruturada, buscava-se evoluir seus mecanismos, para garantir a qualidade dos serviços prestados. Esse processo foi marco importante para a elaboração dos novos contratos, buscando uma maior eficiência, para que pudesse beneficiar tanto o poder público quanto a iniciativa privada, possibilitando maiores benefícios ao consumidor (RIBEIRO; MEYER, 2006).

Para que o contrato de concessões se tornasse uma ferramenta viável para estados e municípios, em 1996 foi promulgada a Lei Federal no 9.277[4], a qual permitia a solicitação da delegação de trechos federais, para que esses entes pudessem fazer parte de seus regimes de concessão rodoviária. Ainda em 1996, a União e o Estado do Rio Grande do Sul firmaram convênios de delegação de rodovias federais, os quais viriam a integrar o PECRRS, com vigência de 15 anos. Quanto à elaboração dos contratos, um ponto importante é que a Agência Estadual de Regulação dos Serviços Públicos Delegados do Rio Grande do Sul(AGERGS), agência reguladora que cuida deste tipo de serviço no estado, começou a operar em 1997, sendo que os primeiros contratos foram celebrados em 1998 (RIO Grande do SUl, 2009; Souza JR.; BalbinotTo Neto, 2005).

Em particular, o governo gaúcho trabalhou com a metodologia de subsídios cruzados, baseando-se na proposta do Departamento Autárquico de Estradas de Rodagem (Daer) no agrupamento de trechos de maior tráfego com os de menor tráfego. Essas passagens eram selecionadas devido à sua importância econômica, além daquelas que necessitavam investimentos para a melhoria da segurança e conforto ao usuário final, visto que na época os investimentos apresentavam-se de forma deficitária (ROSA et al., 2010; RECK, 2012).

No sentido de avaliar as concessões federais e as do Rio Grande do Sul, Profeta et al. (2015) observaram que o modelo gaúcho apresentava pouca clareza em relação a quais padrões seriam seguidos, além de não terem sido impostas definições sobre quais parâmetros seriam usados para avaliar a qualidade do serviço prestado pelo concessionário. Outra questão apontada pelos autores é a existência de assimetria de informações, o que 
pode ocasionar em risco moral, visto que o regulador possui conhecimento limitado em relação ao mercado no qual se está regulando, o que pode gerar o chamado comportamento oportunista por parte da empresa prestadora do serviço.

Por fim, em um estudo mais recente sobre o tema, Pivatto et al. (2017) investigaram as rodovias da terceira etapa de concessões rodoviárias federais, com a realização de simulações para os trechos dessa etapa, além de uma simulação para uma empresa representativa do setor. Nos excertos apresentados foram celebrados contratos de 30 anos de duração (apenas uma exceção, que foi de 25 anos), podendo ser renovados uma vez por igual período. No entanto, foi observado que, mesmo os contratos tendo sido assinados há poucos anos (a partir de 2013), foi possível inferir que há possibilidade de que alguns dos contratos venham a apresentar desequilíbrios dentro de sua execução, inclusive com pedidos de refinanciamento.

\section{Modelo de simulação}

A preocupação com a eficiência da prestação dos serviços, bem como com possíveis desequilíbrios econômicos e financeiros das concessões estaduais, não é uma temática recente, mas o assunto segue em voga. Neste sentido, Paranaíba et al. (2016) relatam que as projeções de fluxo de veículos podem ter resultados espúrios, o que pode ocasionar eventuais comportamentos oportunistas por parte do licitante vencedor.

Tendo em vista as incertezas dos projetos de longo prazo, já que, quanto maior o tempo de contrato, mais difícil é realizar previsões precisas acerca do cumprimento do mesmo; o procedimento de Monte Carlo pode ser utilizado como um instrumento para simular diversos cenários possíveis considerando-se que esse mecanismo possui um baixo custo computacional (MALINI, 1999; NG et al. (2007); NG; XIE, 2008).

Com o propósito de verificar qual o tempo de duração ótimo de um contrato na modalidade de Parceria Público Privada (PPP), Ng et al. (2007) propuseram um modelo de simulação contratual de Monte Carlo. No algoritmo proposto pelos pesquisadores, o período do contrato de concessão é um parâmetro de output (saída) e não de entrada. 
Ao inserirem-se as informações sobre a taxa interna de retorno e sobre o regime tarifário, cada ciclo pode ser calculado de acordo com a estrutura de custos e receitas.

O algoritmo computacional proposto por esses autores utiliza diversos dados para estimar um tempo ótimo de contrato. Entre essas informações aplicadas ao trabalho dos mesmos estão a tarifa cobrada pela concessionária, o investimento feito nos cinco primeiros anos, o custo operacional, o fluxo de veículos, a taxa de crescimento de veículos e as taxas internas de retorno (TIRs), para que com estas se compare a probabilidade de o projeto, ao menos teoricamente, estar dentro do prazo de contrato. O Quadro 1 expressa o algoritmo de simulação proposto pelos pesquisadores:

\section{Quadro 1 | Diagrama de simulações para determinar o período de concessão}

\begin{tabular}{|c|c|}
\hline Etapa 1 & Inicia-se o ciclo de simulação, (total 1000 réplicas) dados $t=0$ e VPL =0. \\
\hline Etapa 2 & $\begin{array}{l}\text { Seja } t=t+1 \text {. } \\
\text { 1. Insere-se os parâmetros iniciais do contrato. } \\
\text { 2. Gera-se números aleatórios de uma distribuição normal para determinar os } \\
\text { valores da taxa de inflação. } \\
\text { 3. Calcula-se, ou determina-se, o custo (inicial) no ano } t, C_{t^{\prime}} \text { de acordo com os } \\
\text { valores da taxa de inflação gerados no passo anterior. } \\
\text { 4. Desconta-se } C_{t} \text { para calcular o VPL utilizando a taxa de retorno esperada. }\end{array}$ \\
\hline Etapa 3 & $\begin{array}{l}\text { Tem-se } t=T_{c} \text { (período de construção)? Se sim, prossegue-se para a Etapa 4. Caso } \\
\text { contrário, volta-se à Etapa } 2 .\end{array}$ \\
\hline Etapa 4 & $\begin{array}{l}\text { Seja. } t=t+1 \\
\text { 1. Gera-se números aleatórios de uma distribuição normal para determinar os } \\
\text { valores dos riscos (crescimento do tráfego de veículos). } \\
\text { 2. Calcula-se, ou determina-se, o custo (inicial) no ano } t, C_{t^{\prime}} \text { de acordo com os } \\
\text { valores de risco gerados no passo anterior. } \\
\text { 3. Desconta-se } C t \text { para calcular o VPL usando a taxa de retorno esperada. }\end{array}$ \\
\hline Etapa 5 & $\begin{array}{l}\text { Gera-se números aleatórios de uma distribuição normal para determinar a } \\
\text { quantidade de usuários do ano } t \text {; } \\
\text { Regime de tarifas quantidade de usuários }=>\text { Receita operacional, } R_{\mathrm{t}} \text {. }\end{array}$ \\
\hline Etapa 6 & $\begin{array}{l}\text { 1. Calcula-se a renda do ano } t: I_{t}=R_{t}-C_{t} \text {. } \\
\text { 2. Desconta-se } I t \text { para calcular o } V P L \text { utilizando a taxa de retorno esperada. }\end{array}$ \\
\hline Etapa 7 & $\begin{array}{l}\text { O } V P L \geq 0 \text { ? Se sim, prossegue-se à Etapa } 8 \text {. Caso contrário, volta-se à Etapa } 4 \text { e } \\
\text { repete-se a operação até que se consiga ao menos um } V P L \geq 0 \text {, para que se possa } \\
\text { seguir à Etapa } 8 \text {. }\end{array}$ \\
\hline Etapa 8 & O período de concessão é $t$. \\
\hline Etapa 9 & O ciclo se encerra. \\
\hline
\end{tabular}

Fonte: NG et al. (2007) (adaptado). 


\subsection{Parâmetros do modelo}

O modelo de simulação a ser executado possui um número de parâmetros que fornecem a calibragem inicial. Abaixo, listam-se os componentes básicos, como segue:

1. Período de Manutenção $\left(\mathbf{M}_{c}\right)$ : trata-se do período de duração do contrato propriamente dito, partindo do tempo de operacionalização e manutenção do mesmo.

2. Taxa Interna de Retorno (TIR): é a taxa mínima atribuída pelo investidor, para que o projeto possa ser viável economicamente. A TIR é a taxa que torna o VPL (valor presente líquido) igual a zero, na série de fluxos de caixa da empresa. Ainda devido às incertezas que o projeto apresenta, o investidor possui uma taxa de retorno mínima, para poder realizar o projeto.

3. Regime de tarifas: o regime de tarifas é baseado na média das tarifas cobradas historicamente no programa, para que se faça uma análise do mesmo.

Além de parâmetros iniciais, o modelo também pode admitir, por exemplo, a existência da inflação no período de análise, visto que é uma característica natural da economia. Na sequência, tem-se os parâmetros que sofrem influência de fenômenos puramente econômicos ou financeiros:

1. Custo no ano $\mathbf{t}\left(\mathbf{C}_{t}\right)$ : considera-se o custo total do projeto, ou seja, incluindo custos de elaboração do projeto, operacionalização, gerenciamento, manutenção, gastos com pessoal, dentre outros.

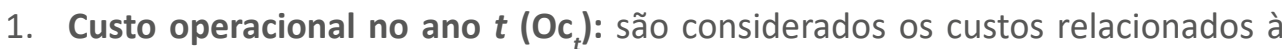
administração da concessionária e a operação do polo.

1. Receita operacional em $\boldsymbol{t}\left(\mathbf{R}_{t}\right)$ : a receita deste tipo de contrato é gerada pela utilização dos serviços por parte do consumidor final, sendo representada pelo número de usuários e pelo regime de tarifas estabelecido. Enquanto o regime de tarifas é um parâmetro determinístico, o número de usuários pode variar de acordo com o nível de desenvolvimento econômico do lugar onde se instala a 
praça do pedágio, a disposição a pagar dos consumidores, a existência de rotas alternativas, dentre outros.

2. Renda em $t\left(I_{t}\right)$ : a renda anual no período operacional é representada pela diferença entre a receita e o custo total no correspondente ano. Após o período de construção, a renda é a diferença entre a receita operacional e o custo operacional.

Ainda, devem ser consideradas algumas hipóteses adicionais para a execução da simulação. A primeira delas é que o cronograma de investimentos segue a proporção de: $10 \%, 20 \%, 30 \%, 20 \%$ e $20 \%$, para os primeiros cinco anos, a começar pelo segundo ano do contrato. Por simplificação, admite-se que haja um período de construção, de 5 anos, dedicado a obras de recuperação, manutenção das vias, dentre outros. Adicionalmente, supõe-se que a economia apresente uma taxa de inflação seguindo uma distribuição normal com média de 0.05 e um desvio padrão de 0.2. O volume de tráfego também apresenta uma taxa de crescimento (ou decrescimento) com base num desvio padrão de $20 \%$. Por fim, o custo operacional segue uma distribuição uniforme definida no intervalor [COR-0.02, COR+0.02], em que COR é a taxa média do custo operacional no período de concessão como uma proporção das receitas.

\subsection{Dados}

Para a realização deste estudo, levantaram-se os dados referentes ao período de concessão tendo como base os editais de licitações de sete trechos, a saber: Polo Metropolitano (Porto Alegre - Metrovias), Polo Gramado (Convias), Polo Vacaria (Rodosul), Polo Caxias do Sul (Brita), Polo Carazinho (Coviplan), Polo Santa Cruz do Sul (Santa Cruz) e Polo Lajeado (Sul Vias). Nesses documentos destacam-se que as companhias vencedoras foram as que ofereceram uma proposta que abrangesse o gerenciamento de uma maior extensão rodoviária (quilometragem) com a cobrança de uma tarifa mais baixa de pedágio.

Um ponto importante a ser considerado é que o volume total de veículos não está separado por categoria, desta forma utilizou-se a tarifa média inicial para se conseguir projetar as receitas futuras. Ainda, o volume inicial de veículos foi considerado no período de 1999, tendo em vista que todas as empresas já estavam operando neste ano e que para 
a maioria das concessionárias a variação do fluxo de veículos entre o primeiro e o segundo ano é bastante elevada.

Na Tabela 1, apresentam-se os dados utilizados para as simulações. Cabe destacar que o custo operacional está apresentado como uma proporção das receitas totais.

Tabela 1 | Resumo dos dados utilizados

\begin{tabular}{lccccc}
\hline Empresa & $\begin{array}{c}\text { Custo } \\
\text { operacional } \\
\text { (\%) }\end{array}$ & $\begin{array}{c}\text { Cresc. do } \\
\text { fluxo de } \\
\text { veículos (\%) }\end{array}$ & $\begin{array}{c}\text { Tráfego inicial } \\
\text { (milhões de } \\
\text { veículos) }\end{array}$ & $\begin{array}{c}\text { Preço inicial } \\
\text { (R\$) }\end{array}$ & $\begin{array}{c}\text { Investimento } \\
\text { inicial } \\
\text { (milhões R\$) }\end{array}$ \\
\hline Metrovias & 24 & 10 & 4,62 & 4,16 & 74,67 \\
\hline Brita & 34 & 13 & 1,072 & 3,74 & 14,44 \\
\hline Sul Vias & 21 & 18 & 4,516 & 4,38 & 59,68 \\
Coviplan & 21 & 22 & 2,681 & 4,53 & 45,22 \\
Rodosul & 27 & 12 & 1,239 & 5,51 & 26,25 \\
Convias & 30 & 21 & 3,000 & 3,46 & 33,91 \\
\hline Santa Cruz & 21 & 10 & 2,256 & 3,78 & 43,85 \\
\hline
\end{tabular}

Fonte: elaboração própria com base em PECR-RS.

Com base nos dados da Tabela 1, é importante relatar que o crescimento do tráfego de veículos foi considerado como a taxa média de crescimento do segundo período até o último. A mesma simplificação foi utilizada para o cálculo da proporção média dos custos operacionais sobre as receitas. Esse procedimento foi feito porque a variação entre o primeiro período e o segundo é muito abrupta, fazendo com que o valor médio ficasse discrepante do valor real ao longo do tempo de operação das empresas. Nesse sentido, como o preço médio inicial da empresa Brita era muito baixo, $\mathrm{R} \$ 0,80$, o que inviabilizaria as simulações, considerou-se o preço médio inicial do segundo período de operação.

Em relação ao investimento, considera-se o valor investido para reparo e ajustes no trecho operado apenas nos cinco primeiros anos. Em geral, nesse período a empresa concessionária dispende um maior valor monetário para melhorias e ajustes na rodovia. 


\section{Resultados}

Inicialmente, fez-se uma simulação para cada um dos sete trechos contratados no Programa de Concessões Rodoviárias, os quais foram calculados separadamente. O fluxo para um período de doze meses de cada modalidade de veículo e o preço inicial também foram realizados para cada tipo de veículo.

Adicionalmente deve-se destacar que cada polo trabalhou com uma TIR diferente. Um aspecto interessante a ser considerado é que, em virtude da situação econômica daquele período, caracterizada por bastantes incertezas, as TIRs trabalhadas foram bastante elevadas, se comparadas com investimentos realizados atualmente.

Pivatto et al. (2017) observam que, para fins de comparação, a TIR utilizada nos contratos de concessão da terceira etapa de concessões federais era de 7,2\%, contra taxas que poderiam passar de $20 \%$ ao ano, devido ao risco inerente ao projeto. Na Tabela abaixo, tem-se as TIRs projetadas para cada empresa participante do certame, de acordo com as suas propostas comerciais (PC) e nos projetos básicos de exploração (PBE).

Tabela 2 | Comparativo entre PBE e PC - Taxa Interna de Retorno

\begin{tabular}{lccc}
\hline $\begin{array}{l}\text { Polo rodoviário } \\
\text { (empresa) }\end{array}$ & $\begin{array}{c}\text { PBE (sem financ. \%) } \\
\text { (TIR 1) }\end{array}$ & $\begin{array}{c}\text { PC (sem financ. - \%) } \\
\text { (TIR 2) }\end{array}$ & $\begin{array}{c}\text { PC (com financ. - \%) } \\
\text { (TIR 3) }\end{array}$ \\
\hline Metrovias & 16,30 & 20,43 & 26,10 \\
\hline Brita & 15,90 & 20,07 & 27,69 \\
\hline Sul Vias & 17,00 & 18,70 & 23,60 \\
Coviplan & 17,60 & 21,77 & 28,12 \\
Rodosul & 17,40 & 18,52 & 27,16 \\
Convias & 16,30 & 17,60 & 24,34 \\
Santa Cruz & 17,20 & 18,30 & 23,32 \\
Média & 16,81 & 19,34 & 25,76 \\
\hline
\end{tabular}

Fonte: PECR-RS.

Como é possível observar na Tabela 2, as TIRs apresentadas pelas empresas, tanto nas propostas comerciais (PC) quanto nos projetos básicos de exploração (PBE) são bastante elevadas, em virtude dos riscos contratuais e conjunturais. Quanto às propostas 
sem e com financiamento, esta última, se aprovada, seria dada pelo Banco Nacional de Desenvolvimento Econômico e Social (BNDES)(RIo GRANDE Do SUL, 2009). Reck (2012) destaca que as variáveis fixas que foram expostas no PBE foram: i) valor da tarifa, ii) tempo de concessão e iii) trechos de conservação obrigatória.

Assim, na Tabela 3, resumem-se os resultados das simulações com base nos parâmetros apresentados na Tabela 1 e nas TIRs mostradas acima. Avalia-se o andamento do programa com os preços iniciais utilizados, considerando uma taxa de inflação de $5 \%$ ao ano, para que se possa corrigir possíveis acréscimos nos preços das tarifas.

Tabela 3 | Cenários simulados

\begin{tabular}{|c|c|c|c|c|c|c|c|c|c|}
\hline \multicolumn{4}{|l|}{ TIR1 } & \multicolumn{3}{|l|}{ TIR2 } & \multicolumn{3}{|l|}{ TIR3 } \\
\hline Praça & $\begin{array}{l}\text { VPL } \\
\text { (R\$ mi) }\end{array}$ & $\begin{array}{l}\text { Tempo } \\
\mathrm{p} / \mathrm{VPL} \\
\geq 0 \text { (em } \\
\text { anos) }\end{array}$ & $\begin{array}{l}\text { Prob } \\
(\mathrm{T} \leq \mathrm{t})\end{array}$ & $\begin{array}{l}\text { VPL } \\
\text { (R\$ mi) }\end{array}$ & $\begin{array}{l}\text { Tempo } \\
\mathrm{p} / \mathrm{VPL} \\
\geq 0 \text { (em } \\
\text { anos) }\end{array}$ & $\begin{array}{l}\text { Prob } \\
(\mathrm{T} \leq \mathrm{t})\end{array}$ & $\begin{array}{l}\text { VPL } \\
\text { (R\$ mi) }\end{array}$ & $\begin{array}{l}\text { Tempo } \\
\mathrm{p} / \mathrm{VPL} \\
\geq 0 \\
\text { (em } \\
\text { anos) }\end{array}$ & $\operatorname{Prob}(\mathrm{T} \leq \mathrm{t})$ \\
\hline Metrovias & 3.97 & 12 & 0.75 & 0.88 & 13 & 0.62 & 0.38 & 18 & 0.91 \\
\hline Brita & 3.16 & 13 & 0.98 & 0.28 & 15 & 0.99 & 0.39 & 19 & 0.93 \\
\hline Sul Vias & 3.09 & 11 & 0.99 & 8.68 & 12 & 0.99 & 0.48 & 12 & 0.92 \\
\hline Coviplan & 5.80 & 12 & 0.99 & 0.16 & 14 & 0.99 & 3.98 & 15 & 0.99 \\
\hline Rodosul & 1.76 & 15 & 0.99 & 3.64 & 14 & 0.98 & 0.03 & 15 & 0.43 \\
\hline Convias & 6.70 & 12 & 0.99 & 5.70 & 12 & 0.99 & 1.47 & 13 & 0.94 \\
\hline Santa Cruz & 2.75 & 16 & 0.99 & 3.04 & 16 & 0.98 & 4.64 & 15 & 0.32 \\
\hline
\end{tabular}

Fonte: elaboração própria.

Notas: as probabilidades de sucesso referem-se a possível cumprimento do contrato dentro de seu período (no caso, 15 anos).

A Tabela 3 resume os resultados das simulações executadas com base no modelo de Ng et al. (2007). Considerando-se o ordenamento das TIRs apresentado na Tabela 2, na primeira coluna apresenta-se o VPL em milhões de reais; a segunda mostra o tempo para que o valor presente líquido fosse maior que zero; e a terceira apresenta a probabilidade de o tempo de concessão ser menor ou igual ao valor da segunda coluna. Essa probabilidade foi estimada usando a função de distribuição empírica das réplicas de Monte Carlo. 
Tendo como base os parâmetros estipulados, com a primeira TIR todos os polos conseguem obter um VPL maior que zero. Pode-se destacar que somente a concessionária Rodosul atinge esse valor no período de 15 anos, e a Santa Cruz precisa de um ano adicional para obter essa marca. Ainda a empresa Sul Vias obtém essa métrica no período de 11 anos, ou seja, com quatro anos de antecedência.

Para a segunda taxa interna de retorno, novamente salvo o trecho administrado pela empresa Santa Cruz, todas as demais conseguiriam obter um VPL maior que zero dentro do prazo contratual estipulado. Destaca-se o caso da empresa Sul Vias, que apresentou um grande montante para o valor presente líquido comparada às demais e um período de três anos inferior ao estabelecido contratualmente para o cumprimento dessa marca.

Avaliando-se a TIR3, observa-se que em média os VPLs são menores, isto é, com o acréscimo da TIR a probabilidade que o VPL seja maior que zero no período de 15 anos é menor. Nesse cenário, destacam-se os casos da Rodosul, que possui apenas $43 \%$ de chances de que o valor presente líquido seja maior que zero nesse período e o da Santa Cruz, que apresenta somente $32 \%$ de chances de sucesso.

De posse dessas informações, observa-se que as taxas informadas na Tabela 2 são economicamente viáveis, com algumas exceções, de acordo com o modelo de simulação.

Assim, pode-se notar que, com uma taxa de retorno mais baixa (TIR1), todas as empresas possuem boas chances de obtenção de retorno positivo dentro do prazo contratual estabelecido. Contudo, ao considerarem-se as incertezas de política econômica, sendo essas incorporadas no valor da taxa interna de retorno, as chances de sucesso do cumprimento contratual dentro do prazo estipulado diminuem de forma drástica apenas para os trechos das concessionárias Santa Cruz e Rodosul.

Embora as estimativas sejam restritivas, devido à parametrização imposta pelo modelo de simulação, os resultados indicam que, com as taxas internas apresentadas nos projetos básicos de exploração há uma boa chance de cumprimento correto do certame, sem a necessidade de renegociações e aditivos contratuais. No entanto, quando são apresentados os cenários com a proposta comercial e a TIR3 os trechos concessionados pela empresa Rodo Sul e Santa Cruz apresentam-se economicamente inviáveis. 
Como uma forma de se verificarem a estabilidade das simulações, realizou-se uma análise de sensibilidade osciland o o valor da TIR1 até a TIR3 em 0.01. A Tabela 4 apresenta as médias para as variáveis computadas, como segue:

Tabela 4 | Análise de sensibilidade para a TIR

\begin{tabular}{lcccc}
\hline Praça & VPL (R\$ mi) & $\begin{array}{c}\text { Tempo } p / \text { VPL } \geq 0 \\
(\mathrm{em} \text { anos) }\end{array}$ & $\begin{array}{c}\text { Probabilidade } \\
(\mathrm{T} \leq \mathrm{t})\end{array}$ & TIR \\
\hline Metrovias & 3.95 & 15 & 0.84 & 0.21 \\
Brita & 0.78 & 15 & 0.80 & 0.22 \\
Sul Vias & 5.78 & 12 & 0.94 & 0.20 \\
Coviplan & 3.84 & 13 & 0.92 & 0.23 \\
Rodosul & 1.55 & 14 & 0.79 & 0.22 \\
Convias & 2.76 & 12 & 0.91 & 0.20 \\
Santa Cruz & 1.92 & 16 & 0.81 & 0.20 \\
\hline
\end{tabular}

Fonte: elaboração própria.

Tomando como referência a análise de sensibilidade, nota-se que, para uma pequena variação (de 0.01 pontos) entre a TIR1 e a TIR3, em média as concessões seriam cumpridas dentro do prazo de 15 anos. É importante enfatizar que as concessionárias Sul Vias, Coviplan, Rodosul e Convias conseguiriam obter um VPL positivo antes do término do certame. Somente a empresa Santa Cruz precisaria de um ano adicional.

De acordo com os estudos prévios de Souza Jr e Balbinoto (2005), Rosa et al. (2010), Reck (2012), Profeta et al. (2015), Parnaíba et al. (2016), essas diferenças entre o período de contrato e a obtenção de um retorno financeiro positivo por parte da maioria das concessionárias nos trechos avaliados, podem se dar principalmente por assimetrias informacionais. Em resumo, se as firmas conseguiram prever adequadamente o montante de custos atrelados ao longo do período de contrato e mensuraram corretamente as possíveis oscilações econômicas, as mesmas podem ter se beneficiado da administração do empreendimento. Deste modo, o papel do governo como prestador de serviços à população é de fiscalizar e estabelecer métricas para computar se não há abuso por parte das firmas (por exemplo, lucros extraordinários) e se o consumidor está sendo atendido de forma adequada. 
Em suma, embora os resultados apresentem a adequabilidade das concessões estaduais, deve-se atentar para algumas restrições do modelo de simulação: (i) não são controlados os possíveis desdobramentos jurídicos por parte tanto do Poder concedente quanto do Judiciário que poderiam promover mudanças ou aditivos contratuais; (ii) o cálculo da taxa de crescimento dos veículos é realizado sem serem descontados automotores que não pagam tarifa, isto é, não geram receita mas usam o trecho concedido e de certa forma contribuem com os gastos de manutenção; (iii) o uso do preço médio da tarifa não considera os diferentes preços cobrados por veículos leves e pesados o que poderia subestimar as estimativas; (iv) durante esse período houve momentos em que as praças de pedágio foram obrigadas a reduzir suas tarifas ou foram fechadas por decreto do Governo do Estado.

\section{Considerações finais}

Com as necessidades de ampliação da malha rodoviária, um dos instrumentos utilizados pelo governo brasileiro foi o marco regulatório referente à Lei de Concessões. Dentro desse contexto, este artigo teve por objetivo estimar o tempo ótimo dos contratos de concessão de pedágios para o estado, no Rio Grande do Sul, no período de 1998 a 2015. Para realizar tal tarefa usou-se o modelo de simulação proposto por Ng et al. (2007) e dados disponíveis no Relatório de Acompanhamento do Programa Estadual de Concessão Rodoviária do Rio Grande do Sul

Assim, os resultados apontam que, com as taxas internas de retornos propostas no projeto básico de exploração, todas as empresas possuíam uma alta probabilidade do cumprimento contratual dentro do prazo de 15 anos. Destaca-se que algumas delas conseguiriam obter um valor presente líquido positivo antes do período final de concessão. Esse fato pode ser oriundo de boas práticas de governança das firmas, já que o governo deve atuar como fiscalizador para verificar a qualidade das rodovias e coibir a cobrança de taxas abusivas.

Contudo, com as propostas comerciais apresentadas pelas concessionárias Rodo Sul e Santa Cruz os contratos seriam economicamente inviáveis dentro desse período 
de tempo. Como, na época das assinaturas dos contratos, os modelos de concessões rodoviárias eram recentes no Brasil e, de acordo com a instabilidade econômica, os riscos de mercado eram mais elevados, o que demandava que as TIRs fossem mais elevadas do que se tem atualmente. Em outras palavras, quanto mais alta a taxa interna de retorno, mais tempo as concessionárias necessitariam para recuperarem seus investimentos.

Embora o modelo de simulação possua restrições quanto à sua parametrização, o algoritmo apresenta diferenças no tempo assinado contratualmente e no tempo previsto, principalmente quando as taxas de retorno tornam-se mais elevadas. De modo intuitivo, isso quer dizer que não seria possível que as empresas recuperassem o valor investido dentro do prazo estipulado para a concessão.

Por fim, para a evolução deste trabalho, tem-se a possibilidade de construir modelos mais sofisticados, que podem vir com a adição de outros parâmetros referentes às características das próprias empresas e, também, de fatores econômicos que possam tornar a dinâmica ainda mais real e propiciar uma análise ainda mais acurada.

\section{Referências bibliográficas}

Barbo, A. R. de C.; Correia, D. E. R.; Engelmann, E. T.; Guzen, E. R.; Gois, G. de A.; Quebaud, M. R.; ALI, M. M.; SouZA, N. M. de; QuebAUD, S. A evolução da regulação nas rodovias federais concedidas. Revista ANTT, ISSN 2177-6571, v. 2, n.2, novembro.2010.Disponível em: <http:// appweb2.antt.gov.br/revistaantt/ed3/_asp/ed3-artigosEvolucao.asp>. Acessado em 05/11/2019.

Beria, P.; RAmella, F.; LAURINo, A. Motorways economic regulation: a worldwide survey. Transport Policy, n. 41, p. 23-32, 2015.

BiASOTO Jr., Geraldo; Afonso, José Roberto R. Oferta de infraestrutura e desenvolvimento econômico: os desafios do investimento público no Brasil. Brasília, Instituto Brasiliense de Direito Público, Caderno Virtual, v. 2, n. 14, 2007.

Carbonara, N.; Costantino, N.; Pellegrino, R. Concession period for PPP: a win-win model for a fair risk sharing. International Journal of Project Management, v. 32, Issue 7, p. 1223-1332, 2014.

CARPINTERO, S.; BARCHAM, R. Private toll roads: lessons from Latin America. International Conference on Traffic and Transportation Engeneering (ICTTE 2012), IPCSIT v. 26, IACSIT Press, Singapore, 2012.

CONFEDeração Nacional do TRANSPorte (CNT) (2011). Pesquisa CNT de rodovias 2011: relatório 
gerencial. Brasília. CNT:SEST:SENAT, 2011.

DUARTE, A.S.S. Concessões de rodovias federais brasileiras: uma proposta para gestão das receitas extraordinárias a favor da modicidade do pedágio. Dissertação de Mestrado em Transportes, Publicação T.DM - 002a /2012, Departamento de Engenharia Civil e Ambiental, Universidade de Brasília, Brasília, DF, 2012.

GALILEA, P.; MEDDA, F. Does the political and economic context influence the success of a transport project? An analysis of public-private partnerships. Research in Transportation Economics, n. 30, p. 102-109, 2010.

GUASCH, J. L.; LAFFONT, J.J.; StRAUB, S. Renegotiation of concession contracts in Latin America: evidence from the water and transport sectors. International Journal of Industrial Organization, n. 26, p. 421-442, 2008.

MALINI, E. Build Operate Transfer Municipal Bridge Projects in India. Journal of Management in Engeneering, n. 15, p. 51-58, 1999.

NG, S. T.; CheUnG, Y. K.; Jefferies, M.; XIE, J. A simulation model for optimizing the concession period of public-private partnerships schemes. International Journal of Project Management, $\mathrm{n}$. 25, p. 791-798, 2007.

ParanaíBA, A.D.C; FonsecA, A.P.; CostA, A.D.J.B. Review and suggestions to the demand forecast model in highway concessions projects in Brazil. Journal of Research in Economics and International Finance, v. 5, n. 1, p. 8-13, 2016.

Pivatto, D.; Fernandez, R. N.; Saulo, H.; Carraro, A. Estimating the Optimal Time for a Road Concession Contract in Brazil. International Journal of Economics and Finance, v. 9, n. 12, 2017.

PINHEIRO, A. C. Regulatory reform in Brazilian infrastructure: where do we stand? Texto para discussão no 964, Ipea, 2003.

Profeta, G. A.; Lírio, V. S.; SAntos, E. S. Modelos de concessões de rodovias no Brasil e necessidade de regulação no setor. Revista Espacios, v. 36, n. 16, 2015.

RECK, D. C. S. O Estado como regulador econômico: uma avaliação da experiência gaúcha de concessão de rodovias (dissertação de mestrado). Programa de Pós-Graduação em Economia, Mestrado em Economia do Desenvolvimento, Pontifícia Universidade Católica do Rio Grande do Sul (PUC-RS), 2012.

RiBeIRo, M. P.; MeYer, A. Private Participation in Infrastructure - the legal and regulatory frameworks in the case of Brazil. OECD Investment Committee "International Investor Participation in Infrastructure: Lesson for Governments", Paris, 2006.

Rio Grande do Sul. Agência Estadual de Regulação dos Serviços Públicos Delegados do Rio Grande do Sul (Agergs). Programa Estadual de Concessão Rodoviária - histórico, evolução e desequilíbrios contratuais - Análise descritiva - 1996/2009, 2009. Disponível em: www.agergs. rs.gov.br. Acessado em 20/09/2017.

RIO GRANDE Do SUl. Departamento Autônomo de Estradas de Rodagem (Daer). Relatório de acompanhamento do Programa de Concessões de Rodovias no Rio Grande do Sul - 1998 a 2012. 
Relatório n. 27, (2013). Disponível em: www.daer.rs.gov.br. Acessado em 20/09/2017.

RosA, M. V. F. DA.; SenNA, L. A. Dos S.; LindAU, L. A. Highways concessions - Brazilian View. $12^{\text {th }}$ WCTR, July 11-15, 2010 - Lisbon, Portugal.

SouZA JR., R. T. de.; BALBINOTto Neto, G. O leilão de Demsetz como mecanismo regulador: a experiência gaúcha na concessão de rodovias. IV Congresso Brasileiro de Regulação, Manaus, Abar, junho de 2005.

\section{Rodrigo Nobre Fernandez}

(iD) https://orcid.org/0000-0001-8596-2898

Doutor em Economia pelo PPGE/UFRGS. Professor do Departamento de Economia da Universidade Federal de Pelotas.

E-mail:rodrigo.fernandez@ufpel.edu.br

\section{André Carraro}

(D) https://orcid.org/0000-0001-5085-8185

Doutor em Economia pelo PPGE/UFRGS. Professor do Departamento de Economia da Universidade Federal de Pelotas.

E-mail: andre.carraro@gmail.com

\section{Helton Saulo}

https://orcid.org/0000-0002-4467-8652

Doutor em Economia pelo PPGE/UFRGS. Professor do Departamento de Estatística da Universidade de Brasília.

E-mail: heltonsaulo@gmail.com

\section{Douglas Pivatto}

(D) https://orcid.org/0000-0001-6759-2694

Mestre em Economia Aplicada pela Universidade Federal de Pelotas.

Servidor da Prefeitura Municipal do Rio Grande.

E-mail: douglaspivatto@hotmail.com

\section{Everton Freitas}

(D) https://orcid.org/0000-0002-2442-214X

Bacharel em Engenharia de Produção pela Universidade Federal de Pelotas. E-mail: everton_freitas1996@ hotmail.com

\section{Agradecimentos}

Rodrigo Nobre Fernandez gostaria de agradecer ao Conselho Nacional de Desenvolvimento Científico e Tecnológico (CNPq) pelo suporte financeiro.

Material Suplementar:

O material suplementar, bem como, planilhas e editais e scripts usados para realizarem-se as simulações podem ser obtidos em: http://www.rodrigofernandez.com.br/mat_sup_enap.zip 


\section{Apêndice 1}

\# Versao executada no R-3.6.1

\# Pacotes necessarios

\# Se voce nao tiver e necessario realizar a instalacao

\# Para isso rode o seguinte comando: install.packages("nome_do_pacote")

\# Exemplo:install.packages(“FinCal”)

\#Limpar o espaco de trabalho

$\mathrm{rm}($ list $=\mathrm{Is}())$

\#Carregando as bibliotecas (pacotes) necessárias

library(FinCal)

library(Hmisc)

library(EnvStats)

\# Simulacao de Monte Carlo - Fatores Aleatórios

\# Valor Presente Liquido $(\mathrm{VPL})=$ Net Present Value (NPV)

\# Os dados dessa simulacao estao calibrados para o polo metropolitano

\# concessionaria Metrovias

NREP <- 1000 \# Replicas de Monte Carlo

Tc <- 6 \# Tempo de Contrucao (considera-se 5 anos)

apportioned <- c $\quad(0.0,0.1,0.2,0.3,0.2,0.2) \#$ estrutura de custos no periodo de construcao 
vecIRR2

$<-c(0.163,0.2043,0.261)$

\#vecIRR

$<-0.261$

\# Analise de Sensibilidade

veclRR<-seq(veclRR2[1],veclRR2[3],by=0.001) \# tirar o comentario para realizar a para a analise de sensibilidade

$$
\begin{aligned}
& \text { prob1<-matrix (0,nrow=length(veclRR),ncol=1) } \\
& \text { tempo1<-matrix (0,nrow=length(veclRR),ncol=1) } \\
& \text { npvt<-matrix(0,nrow=length(veclRR),ncol=1) }
\end{aligned}
$$

\#Parametros do modelo

II<- 74676274.37 \# Investimento Incial (5 primeiros anos)

rocost $<-0.24$ \#custo operacional como proporcao das receitas

Ccost <- numeric() \# Custo de Construcao

Ocost <- numeric() \# Custo de Operacao

NPV < - numeric() \# Valor Presente Liquido

Concession_Period <- numeric() \# Periodo de Concessao

Revenue <- numeric() \# Receita Total

Income <- numeric() \# Renda

matrix_NPV <- matrix(NA,NREP,100) \# Essa matriz armazena cada VPL para cada tentativa 
Matrix_Concession_Period <- matrix(NA,NREP,length(vecIRR)) \# Essa matriz armazena o tempo de concessao para cada TIR

Traffic_Volume_cars <- numeric() \# volume de veiculos

Annual_growth_cars <- numeric() \#taxa de crescimento dos veiculos

p_cars <- numeric() \# preco medio por veiculo

First_Traffic_Volume_cars <- 4620869 \# primeiro valor do volume de veiculos ou segundo se a discrepância for muito grande

First_p_cars $\quad<-4.17$ \# preco medio por veiculo no primeiro ano

growth_cars <- $(1+0.1)$ \# taxa de crescimento médio no período de concessão

\# Simulacao de Monte Carlo

for ( $\mathrm{j}$ in 1:length(vecIRR))\{

IRR <- vecIRR[j]

for(i in 1:NREP) \{

t $\quad<-1 \# \mathrm{t}=0$; Primeiro Periodo

$N P V[t]<-0$

Ccost $[\mathrm{t}]<-0$

Total_Construction_Cost <- II

while $(\mathrm{t}<\mathrm{Tc})\{$

$t<-t+1$ 
Ccost <- Total_Construction_Cost * apportioned $*(1+\operatorname{rnorm}(1$, mean $=0.05, \mathrm{sd}=0.02)$ ) \# Custo de Construcao considerando a taxa de inflacao

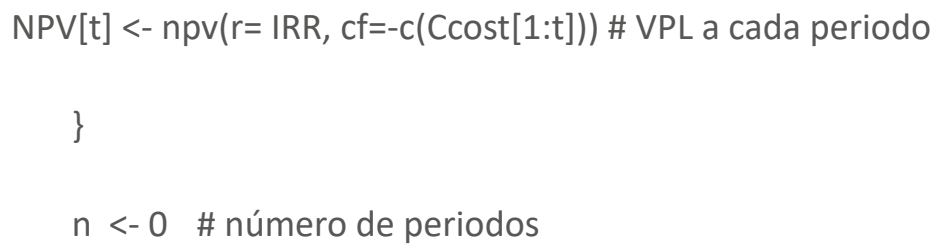


Annual_growth_cars[t] <- Traffic_Volume_cars[t]* ${ }^{*}$ growth_cars $)^{\wedge}(\mathrm{n})$ \# Crescimento anual de carros

Revenue[t] <- p_cars[t]*Annual_growth_cars[t] \# Receita

Ocost[t] <- Revenue[t] $*$ runif(1, $\min =$ rocost-0.02, $\max =$ rocost+0.02)\# Custo Operacional Anual - Flutua dentro de uma distribuicao uniforme

Income[t] <- Revenue[t] - Ocost[t] \# Renda

$\mathrm{NPV}[\mathrm{t}] \quad<-\operatorname{npv}(\mathrm{r}=\mathrm{IRR}, \quad \mathrm{cf}=\mathrm{c}(-$ Ccost,Income[(length(Ccost)+1):t]) ) \# VPL a cada periodo

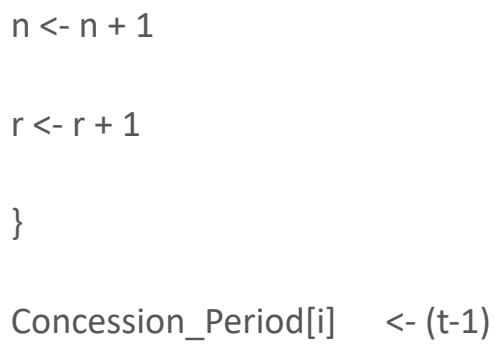

$n p v t[j]<-N P V[t]$

tempo1[j]<-t

matrix_NPV[i,1:length(NPV)] <- NPV

Matrix_Concession_Period[,j] <-Concession_Period \#computa 0 período de concessão para cada TIR 
\# Resultados

npvt <- npvt[!is.na(npvt)]/10^6;

npvt

tempo1 <- tempo1[!is.na(tempo1)];

tempo1

\# Tempo medio de concessao

mCP <-round(mean(Concession_Period));

$\mathrm{mCP}$

\#Tempo de concessao que faz o VPL>0

$\mathrm{t}$

\# VPL

mNPV <- colMeans(matrix_NPV,na.rm=TRUE)/10^6

mNPV <- mNPV[! is.na(mNPV)];

$\mathrm{mNPV}[\mathrm{t}]$ 
\#Grafico do VPL

$x<-$ seq $(1$, length $(m N P V)$, by $=1)$

plot $(x, m N P V, x l a b="$ Periodo de Concessao",ylab="VPL (milhoes)",type $=" \mid ", x \lim =c(0,30))$

abline(h = 0, v = mCP-1, col = "gray60", Ity = 2)

dev.off()

\# Funcao de distribuicao empirica

Concession_Period_IRR001 <- Matrix_Concession_Period[,1]

EmpiricalDist_IRR001 <-Ecdf(Concession_Period_IRR001,pl=FALSE)

\# Ver os resultados

View(EmpiricalDist_IRR001)

for ( $k$ in 1:length(EmpiricalDist_IRR001\$x))

\{

if (EmpiricalDist_IRR001\$x[k]==t)\{

tempo=EmpiricalDist_IRR001\$ $\mathbf{x}[\mathrm{k}]$

prob=EmpiricalDist_IRR001\$̣[ [k] 
\}
\}

\# Resumo dos Resultados

dados $=$ matrix $(c(t$, prob, $m N P V[t])$, nrow $=1$, ncol $=3)$

colnames(dados)=c("tempo","prob","VPL")

dados

\# Para a analise de sensibilidade

for (i in 1:length(veclRR))

\{

Concession_Period_IRR001 <- Matrix_Concession_Period[,i]

EmpiricalDist_IRR001 <- Ecdf(Concession_Period_IRR001,pl=FALSE)

for ( $k$ in 1:length(EmpiricalDist_IRR001\$x))

\{

if (EmpiricalDist_IRR001\$x[k]==tempo1[i]) 


$$
\text { prob1[i]=Empiricaldist_ }
$$

IRR001\$̧y[k]

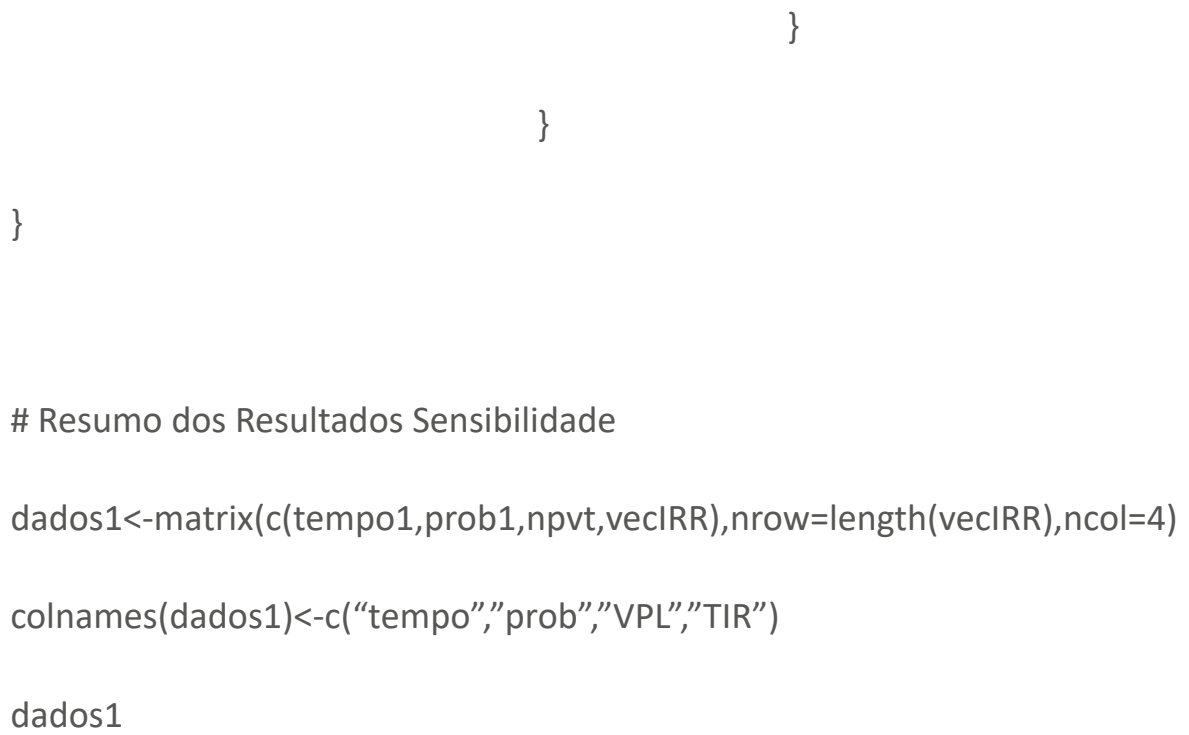

[1] Para mais detalhes veja: http://www.planalto.gov.br/ccivil_03/leis/L8987cons.htm

[2] Veja o texto na íntegra: http://www.planalto.gov.br/ccivil_03/leis//8666cons.htm

[3] https://ppi.worldbank.org/en/ppi

[4] http://www.planalto.gov.br/ccivil_03/leis/L9277.htm 


\section{Anexo 1 - Material Suplementar}

O material suplementar, bem como planilhas e editais, e scripts usados para realizarem-se as simulações podem ser obtidos em:

http://www.rodrigofernandez.com.br/mat_sup_enap.zip

http://www.rodrigofernandez.com.br/script_r.html 